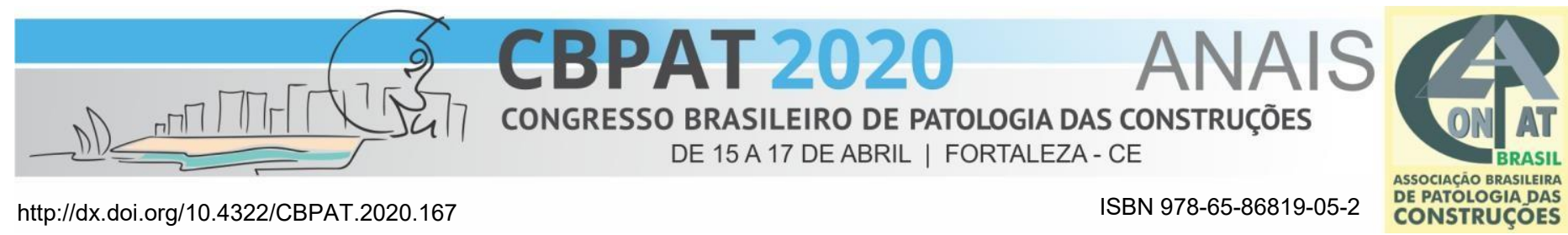

\title{
INFLUÊNCIA DO TEOR DE UMIDADE NO MÓDULO DE ELASTICIDADE DINÂMICO DE CONCRETOS DOSADOS A 30 MPA
}

\author{
PINTO, FRANCISCO DAVI DE LIMA \\ Graduando em Engenharia Civil \\ Faculdade Ari de Sá \\ Ceará; Brasil \\ davilima100@hotmail.com
}

\author{
DE MEDEIROS, JOÃO LUCAS LOPES \\ Graduando em Engenharia Civil \\ Faculdade Ari de Sá \\ Ceará; Brasil \\ joaomedeiros702@gmail.com
}

\author{
MESQUITA, ESEQUIEL FERNANDES TEIXEIRA \\ Professor Doutor em Engenharia Civil \\ Universidade Federal do Ceará \\ Ceará; Brasil \\ mesquita.e@outlook.com
}

\author{
Professora Mestre em Engenharia Civil \\ Ceará; Brasil \\ lilian.brasileiro@outlook.com
}

\section{RESUMO}

A busca por materiais que tenham maior durabilidade e desempenho frente às diversas situações às quais estão expostos tem se tornado objeto de estudo de diversos pesquisadores da tecnologia dos concretos. Tal fato é essencial para um melhor entendimento de como um material pode cumprir suas funções previstas em projeto, sem comprometer assim suas aplicações. Para obter tais resultados, o ensaio de ultrassom mostra-se como uma boa alternativa para uma análise mais precisa das características dos materiais, visto que com o uso do ultrassom é possível estimar a velocidade de propagação da onda ultrassônica e assim determinar possíveis intervenções a serem realizadas em determinada estrutura. Neste cenário, esse trabalho tem o objetivo de verificar a influência do teor de umidade do concreto em medições de velocidade do pulso ultrassônico, medida obtida a partir do ensaio de ultrassom, em corpos de prova cilíndricos $(10 \mathrm{~cm}$ x $20 \mathrm{~cm})$, moldados com concreto de $30 \mathrm{MPa}$ e submetidos à saturação total, considerando o período pós-moldagem de 28 dias. Para a realização desse ensaio não destrutivo, foi seguida a metodologia proposta na NBR 8802 (ABNT, 2019). Os resultados obtidos permitiram observar a influência do teor de umidade nos corpos de concreto, contribuindo assim com o estudo da caracterização do concreto pelo método do Ultrassom.

Palavras-chave: ultrassom, concreto, teor de umidade, módulo de elasticidade dinâmico.

\begin{abstract}
The search for materials that have greater durability and performance in face of the various situations to which they are exposed has become the object of study by several researchers of concrete technology. This fact is essential for a better understanding of how a material can fulfill its intended design functions without compromising its applications. To obtain such results, the ultrasound test proves to be a good alternative for a more accurate analysis of the material characteristics, since with the use of ultrasound it is possible to estimate the propagation velocity of the ultrasonic wave and thus determine possible interventions to be performed in a given structure. In this scenario, this work aims to verify the influence of the moisture content of concrete on ultrasonic pulse velocity measurements, measured from the ultrasound test, in cylindrical specimens $(10 \mathrm{~cm} \times 20 \mathrm{~cm})$, molded with $30 \mathrm{MPa}$ concrete and submitted to total saturation, considering the post-molding period of 28 days. To perform this non-destructive test, the methodology proposed in NBR 8802 (ABNT, 2019) was followed. The obtained results allowed to observe the influence of the moisture content on the concrete bodies, thus contributing to the study of the characterization of the concrete by the ultrasound method.
\end{abstract}

Keywords: ultrasound, concrete, moisture content, dynamic modulus of elasticity. 


\section{INTRODUÇÃO}

Nos últimos anos, aceleraram-se as mudanças tecnológicas voltadas para a área da investigação das possíveis causas de manifestações patologias apresentadas em estruturas de concreto em edifícios. Tal fato é devido à frequência dessas desconformidades nesses elementos, gerando assim diversas interpretações para a real causa dessas desconformidades.

Toda estrutura tem uma vida útil de projeto (VUP) conforme NBR 15.575 (ABNT, 2013), que estabelece critérios e requisitos mínimos de desempenho para edificações, no entanto, para cumprir tais fatores, o edifício deve receber manutenções preventivas para suportar as variáveis do próprio ambiente no qual está inserida.

Dentre as mais diversas manifestações patológicas apresentadas em uma estrutura, deve-se dar destaque às que surgem devido a umidade, na qual acarreta diversos problemas aos sistemas construtivos dos edifícios pelo fato do alto grau de saturação que é desenvolvido. Segundo Parisi Jonov (2013) os problemas de umidade, além de frequentes, representam um dos maiores problemas de uma edificação durante sua vida útil.

Para que uma estrutura possa atingir certo nível de durabilidade e proteção, os sistemas construtivos que o compõem devem receber ações de manutenções preventivas, no qual, os custos gerados para tais intervenções tendem a crescer segundo uma progressão aritmética. Tal afirmação colabora para atender a importância de adoção de políticas de manutenções periódicas de caráter preventivo e/ou preditivo e não por intervenção corretiva, devido ao alto custo empregado que tal intervenção.

O monitoramento constante das estruturas é um procedimento necessário, uma vez que a detecção preventiva dos problemas permite a utilização de métodos mais simples e econômicos para avaliação e reparo (DA, 2019). Os ensaios não destrutivos (END) são grandes aliados para auxiliar nas investigações das causas e até mesmo prever o aparecimento de manifestações patológicas que ainda venham a aparecer e consequentemente prejudicar a estrutura, pois o mesmo tem como base a utilização de equipamentos que não danifiquem a estrutura e possam dar um resultado real do que se pretende analisar.

O uso de métodos de END é uma maneira de viabilizar a inspeção e a avaliação do estado de conservação de construções civis, de forma econômica e eficiente (DA, 2019). O ultrassom é um dos equipamentos de ensaios não destrutivos que utiliza as ondas ultrassônicas para determinar as condições do concreto em cada local específico onde será realizado o ensaio. A NBR 8802 (ABNT, 2013) estabelece procedimentos para a realização de ensaios de velocidade de propagação ultrassônica utilizando esse equipamento. Normalmente, o ensaio de ultrassom é realizado com o objetivo de verificar a uniformidade do concreto, detectar eventuais falhas internas de concretagem, avaliar a profundidade de fissuras ou outras imperfeições, estimar o módulo de deformação e a resistência à compressão e/ou monitorar as variações das características do concreto ao longo do tempo (GOMES, 2016).

Dentre os END, o ultrassom permite a análise durante ou depois da construção, fazendo leituras com rapidez para caracterizar, classificar ou identificar patologias em estruturas de concreto. No entanto, devem ser observados os fatores que influenciam nas leituras, como tipo de agregado, relação água/cimento, umidade, temperatura, presença de armadura e perfeito acoplamento dos transdutores (PAULA, 2018).

A partir desse ensaio, também é possível analisar uma importante propriedade mecânica do concreto que está ligada à sua rigidez, que é o módulo de elasticidade dinâmico. Por apresentar um comportamento não-linear, a curva tensão x deformação do concreto apresenta certa dificuldade para a determinação exata de um único valor do módulo de elasticidade estático, portanto a utilização de métodos experimentais dinâmicos não-destrutivos, que aplicam carregamentos dinâmicos e não interferem diretamente na amostra, fornece o valor do módulo de elasticidade do material de forma mais precisa (COSSOLINO, 2011). 
O presente estudo, tem como objetivo apresentar um estudo minucioso quanto a aplicação de métodos de ensaios não destrutivos em corpos de prova de concreto dosados a $30 \mathrm{MPA}$, colaborando com a determinação da influência do teor de umidade no módulo de elasticidade dinâmico do concreto, mensurado a partir do equipamento de ultrassom, tornando, assim, mais precisas as medições realizadas e gerando assim um campo maior de informações no estudo sobre a influência da umidade no módulo de elasticidade dinâmico e na VPU.

\section{MATERiais e MÉtodos}

\subsection{Materiais}

Os materiais utilizados foram aquelescaracterísticos de um concreto convencional: Cimento Portland CP II-Z-30, com propriedades físicas e mecânicas de acordo com a NBR 16697 (ABNT, 2018) que, segundo informações do fabricante, possui massa específica igual a $3,15 \mathrm{~g} / \mathrm{cm}^{3}$; areia lavada de rio, brita de origem granítica com Dmax igual a $9,5 \mathrm{~mm}$ e água do sistema de abastecimento da cidade de Fortaleza/CE. A Tabela 1 traz alguns dados de caracterização dos agregados.

Tabela 1 - Caracterização dos agregados utilizados na produção do concreto convencional

\begin{tabular}{|l|c|c|}
\hline \multicolumn{1}{|c|}{ Ensaios } & Brita & Areia \\
\hline Massa especifica $\left(\mathrm{g} / \mathrm{cm}^{3}\right)$ & 2,64 & 2,68 \\
\hline Absorção de água $(\%)$ & 0,4 & 0,5 \\
\hline
\end{tabular}

\subsection{Dosagens}

$\mathrm{O}$ concreto foi dosado para atingir uma resistência à compressão axial de $30 \mathrm{MPa}$ aos 28 dias. Foram moldados 12 corpos de prova cilíndricos $(10 \mathrm{~cm} \times 20 \mathrm{~cm}$ ), segundo os parâmetros da NBR 5738 (ABNT, 2015), que após 28 dias de cura imersa em água foram submetidos aos ensaios. Os materiais necessários para a produção dos testemunhos foram aferidos através do suporte de uma balança digital, e posteriormente misturados em betoneira.

\subsection{Cálculo do módulo de elasticidade dinâmico}

Para a análise de deformações e tensões em estruturas carregadas dinamicamente o módulo de elasticidade dinâmico é o mais adequado (MEHTA,1994). Para sua determinação, é feita uma relação entre a velocidade de propagação da onda ultrassônica (VPU) e a densidade do material. Para isso, a Equação 1 é utilizada, seguindo a norma britânica BS - 1881 part 203:1986, semelhante ao que contém na NBR 16530 (ABNT, 2008). A escolha pela norma britânica é devido a maior variedade de aplicações práticas e um maior campo bibliográfico do referido estudo contendo resultados mais concretos de forma a referenciar a norma britânica de forma a possuir um parecer melhor em relação a brasileira.

\section{Onde:}

$$
\mathrm{E}_{\mathrm{d}}=\gamma \cdot \mathrm{v}^{2} \cdot \frac{(1+v) \cdot(1-2 v)}{1-v}
$$

$\gamma=$ densidade do corpo-de-prova;

$\mathrm{V}=$ velocidade de propagação da onda ultrassônica;

$v=$ coeficiente de Poisson (admite-se 0,2 para concreto). 
A referida equação é responsável por nortear os resultados obtidos nos Gráficos 1 e 2, embarcando as características de densidade dos corpos de prova, velocidade de propagação e um coeficiente de Poisson, que aplicados na equação resultam em informações precisas para gerar análises gráficas e assim poder mensurar suas propriedades.

\section{METODOLOGIA}

Para avaliar a determinação da VPU o equipamento utilizado foi o aparelho de ultrassom. Os ensaios de ultrassom foram realizados por transmissão direta, que consiste na emissão de ondas ultrassônica em faces horizontais opostas do testemunho ensaiado, de acordo com a Figura 1.

Figura 1: Metodologia do estudo.
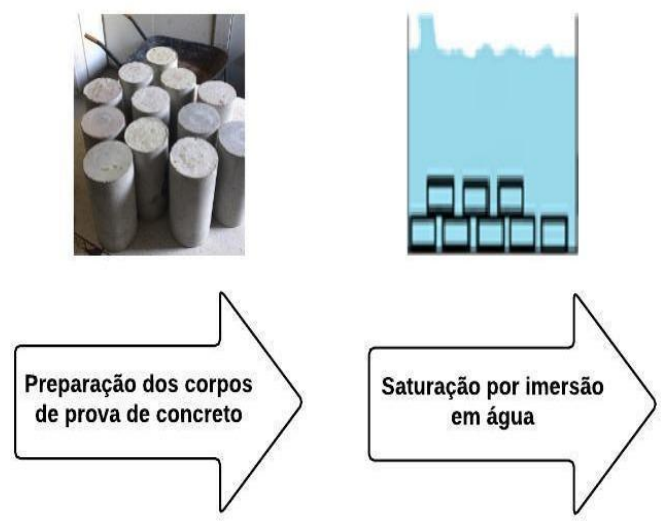

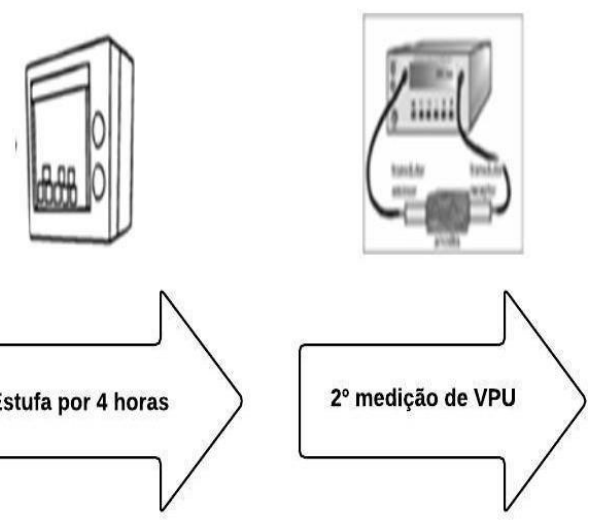

Fonte: autor

Para a realização do ensaio, foi respeitado todos os parâmetros da metodologia Norma Brasileira 8802 (ABNT, 2019) para o ensaio de ultrassom, para assim validar o referido estudo. Para a preparação dos corpos de prova, foi feito todo o tratamento, desde a dosagem até o capeamento e posterior rompimento dos corpos de prova para determinar a resistência à compressão.

A determinação da onda ultrassônica deu-se início primeiramente com a calibragem do aparelho, utilizando uma barra padrão do aparelho, para garantir assim que o ensaio tenha seus resultados precisos. Em seguida, os corpos de provas foram saturados por um período de 24 horas em uma estufa, após o fim do tempo estimado, os corpos de provas foram pesados e realizados exames ultrassônicos, onde foi possível anotar valores para o cálculo do teor de umidade, seguindo os parâmetros da NBR 9778 (ABNT, 2015). Após isso, os elementos de concreto foram levados para estufa com temperatura de $105 \pm, 5^{\circ} \mathrm{C}$, por um período de 4 horas, tempo este suficiente para a secagem, sendo novamente pesados e realizados ensaios para determinação do pulso ultrassônico. O ensaio consistiu, de modo que os testemunhos ensaiados, estivessem totalmente secos, não mais variando seu peso. A Figura 2 ilustra a realização do ensaio de ultrassom nos corpos de prova cilíndricos. 
Figura 2: Transmissão direta do pulso ultrassônico em corpo de prova cilíndrico

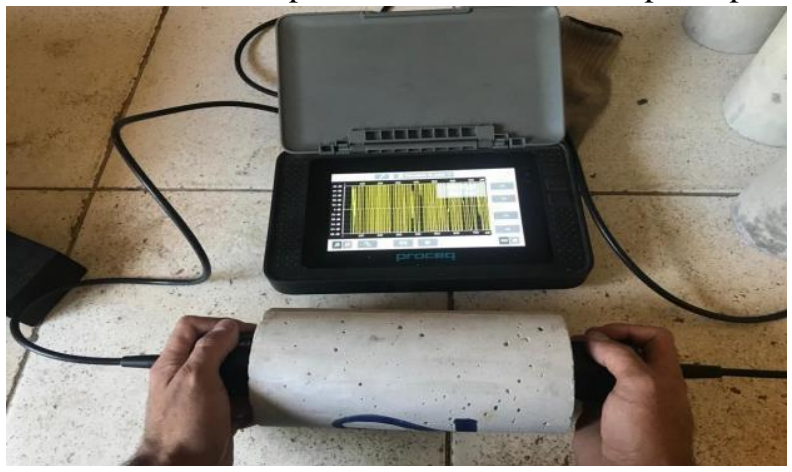

Fonte: autor

\section{Resultados}

Após a análise de toda a metodologia abordada, obtiveram-se os resultados dos corpos de prova cilíndricos com valores de VPU médio de 4194,53 m/s após o período de 28 dias de cura. Com o referido dado, foi possível elaborar gráficos específicos para correlacionar as variáveis apresentadas no VPU de cada corpo de prova, tendo como fator determinante os diferentes teores de umidade. Tal fator foi determinante para a determinação de expressões matemáticas, que são o ponto chave para a aplicação científica desse trabalho, pois a partir deles é possível remover os efeitos da umidade dos valores de VPU e consequentemente do módulo de elasticidade.

A retirada dos valores da umidade por meio de equações possibilita a melhoria da interpretação de resultados, fazendo com que o exame ultrassônico se torne uma ferramenta na qual se obtenham resultados de caracterização físico-mecânico e de caracterização dos materiais, desmitificando o uso de END para inspeções de estruturas e obras de arte.

Após análise dos resultados, foi possível mensurar a correlação entre as variáveis VPU x teor de umidade, constatando que quanto maior a umidade apresentada na amostra dos corpos de prova, maior a velocidade do pulso ultrassônico característico do elemento estudado. Assim como afirma Figueiredo (2005), à medida que os vazios são preenchidos por água, a VPU tende a crescer, como mostra o Gráfico 1 abaixo.

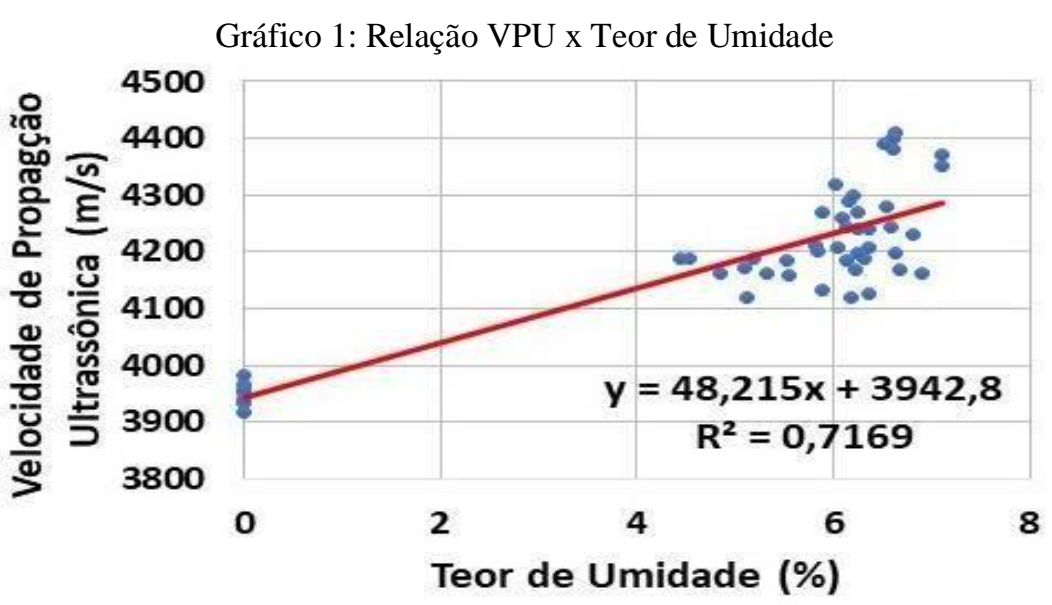


Fonte: autor

Com os dados obtidos, foi possível analisar que a umidade do concreto influencia na velocidade de propagação ultrassônica, tal fato ocasiona um erro na interpretação de como se comporta a estrutura. O Gráfico 2 mostra a relação entre o módulo de elasticidade dinâmico e o teor de umidade.

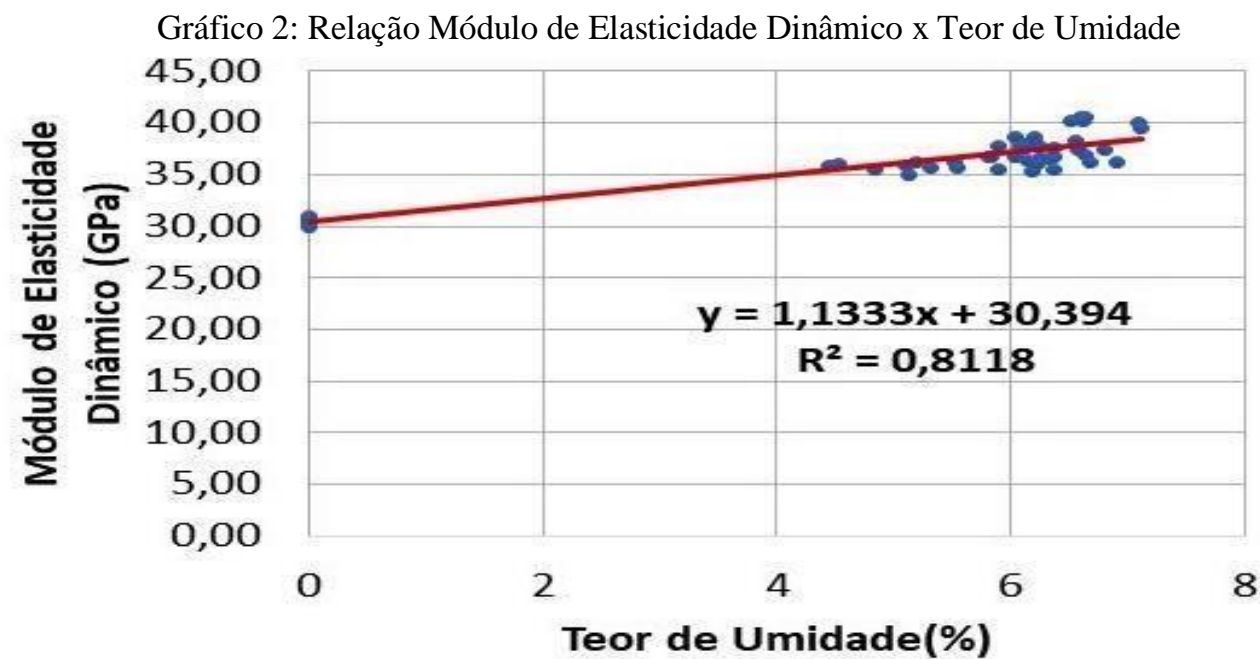

Fonte: autor

A partir da análise do Gráfico 2 é possível observar uma interferência significativa da umidade no módulo de elasticidade, que foi um aumento na ordem de 36\% no valor do módulo, resultando em um acréscimo de 10,5 GPa, algo bastante significante para o concreto. Obtiveram-se coeficientes de determinação $\left(\mathrm{R}^{2}\right)$ bastante confiáveis, mostrando que o presente estudo pode ser utilizado como referência na interpretação de resultados do ensaio de ultrassom.

\section{Conclusão}

O trabalho abordado tem como objetivo analisar de forma precisa a influência da umidade no módulo de elasticidade dinâmico de concretos convencionais dosados a 30 MPA, tendo como motivação para o referido estudo, as crescentes manifestações patológicas apresentadas em estruturas de concreto, nas quais a umidade é uma das principais causas.

O estudo tem como motivação buscar soluções com antecedência para determinar um plano de manutenção e/ou reparação do objeto estudado e assim colaborar em uma mudança benéfica na aplicação políticas de intervenções preventivas e/ou preditivas dentro de um edifício. A pesquisa das causas dos patógenos dessas manifestações é essencial para mitigar fatos distorcidos sobre a real causa das referidas patologias.

Com este estudo apresentado, foi possível observar que o aumento de $7 \%$ de umidade interferiu na elevação de VPU em 12,5\%. Com isso, é possível verificar e mensurar a direta relação entre esses dois fatores. Em relação ao módulo de elasticidade dinâmico, também se notou um aumento com o acrescimento de umidade, resultando em um acréscimo no módulo de 10,5 GPa.

O presente trabalho embarca consigo conceitos correlatos que resultam em situações de estudo aplicáveis para explicar e verificar a influência da umidade no módulo de elasticidade dinâmico de concreto. Com isso, incentivar e explorar estudos nesse campo, investigando o quão esses resultados interferem na estrutura empregada é de fundamental importância para se obter os objetivos apresentados, e assim gerar um campo de visão cada vez maior nessa área de estudo. 


\section{REFERÊNCIAS}

ASSOCIAÇÃO BRASILEIRA DE NORMAS TÉCNICAS. NBR 15630: Argamassa para assentamento e revestimento de paredes e tetos- Determinação do módulo de elasticidade dinâmico através da propagação de onda ultra-sônica. 2008

ASSOCIAÇÃO BRASILEIRA DE NORMAS TÉCNICAS. NBR 8802: Concreto endurecido Determinação da velocidade de propagação de onda ultrassônica. Rio de Janeiro, 2013.

ASSOCIAÇÃO BRASILEIRA DE NORMAS TÉCNICAS. NBR 16697 - Cimento Portland- Requisitos. 2018.

ASSOCIAÇÃO BRASILEIRA DE NORMAS TÉCNICAS. NBR 5738 - Concreto Procedimento para moldagem e cura de corpos de prova. 2015.

ASSOCIAÇÃO BRASILEIRA DE NORMAS TÉCNICAS. NBR 9778 - Argamassa e concreto endurecidos Determinação da absorção de água, índice de vaziose massa específica. 2005.

ASSOCIAÇÃO BRASILEIRA DE NORMAS TÉCNICAS. NBR 15575: Desempenho de edificações habitacionais. Rio de Janeiro. 2013.

BRITISH STANDARD ASSOCIATION. BS 1881 Part 203: Recommendations for measurement of velocity of ultrasonic pulses in concrete. 1986

COSSOLINO, L. C.; PEREIRA, A. H. A.; DEBS, M. K. E. L. Determinação do módulo de elasticidade do concreto a partir da resposta acústica. Volume 11. IBRACON Structures and Materials Journal. 2011.

DA, L. C. P. et al. Estudos de caso sobre avaliação de estruturas de concreto através da utilização de ensaios não destrutivos. Revista ALCONPAT. [s.l: s.n.]. Disponível em: <http://www.mda.cinvestav.mx/alconpat/revista>. Acesso em: 29 jul. 2019.

FIGUEIREDO, E. P. Inspeção e Diagnóstico de Estruturas de Concreto com Problemas de Resistência, Fissuras e Deformações. In: ISAIA, G. C. (Ed.). Concreto: Ensino, Pesquisa e Realizações. São Paulo: IBRACON, 2005

GOMES, C. Avaliação De Pilares De Concreto Armado Através Do Ensaio De Pulso Ultrassônico. Anais do Congresso Brasileiro de Patologia das Construções, p. $1-9,2016$.

MEHTA, P.K.; MONTEIRO, P.J.M. (1994). Concreto: estrutura, propriedades e materiais. São Paulo: Pini.

PARISI JONOV, C. M.; NASCIMENTO, N. O.; PAULO E SILVA, A. Avaliação de danos às edificações causados por inundações e obtenção dos custos de recuperação. Ambiente construído, Porto Alegre, 2013.

PAULA, A.; DOUTORA, P. Variação da velocidade do pulso ultrassônico nas primeiras idades do concreto 1 . I Workshop de Ensaios não-destrutivos aplicados na construção civil, p. 3 , 